\title{
Phase diagram of two-flavor quark matter: Gluonic phase at nonzero temperature
}

\author{
O. Kiriyama* \\ Institut für Theoretische Physik, J.W. Goethe-Universität, D-60438 Frankfurt am Main, Germany \\ and Research Center for Nuclear Physics, Osaka University, Ibaraki 567-0047, Japan
}

(Dated: November 7, 2018)

\begin{abstract}
The phase structure of neutral two-flavor quark matter at nonzero temperature is studied. Our analysis is performed within the framework of a gauged Nambu-Jona-Lasinio model and the meanfield approximation. We compute the free energy of the gluonic phase (gluonic cylindrical phase II) in a self-consistent manner and investigate the phase transition from the gluonic phase to the 2SC/g2SC/NQ phases. We briefly consider the phase diagram in the plane of coupling strength versus temperature and discuss the mixed phase consisting of the normal quark and 2SC phases.
\end{abstract}

PACS numbers: 12.38.-t, 11.30.Qc, 26.60.+c

\section{INTRODUCTION}

The properties of cold and dense quark matter are of great interest in astrophysics and cosmology. In particular, at moderate densities of relevance for the interior of compact stars, quark matter is a color superconductor and has a rich phase structure with important implications for compact star physics [1, 2, 3, 4, 5, 6, 7, 8, 9].

Bulk matter in the interior of compact stars should be color and electrically neutral and be in $\beta$-equilibrium. In the two-flavor case, these conditions separate the Fermi momenta of up and down quarks and, as a consequence, the ordinary BCS state (2SC) is not always energetically favored over other unconventional states. The possibilities include crystalline color superconductivity and gapless color superconductivity (g2SC) [10, 11, 12, 13]. However, the $2 \mathrm{SC} / \mathrm{g} 2 \mathrm{SC}$ phases suffer from a chromomagnetic instability, indicated by imaginary Meissner masses of some gluons [14, 15]. The instability related to gluons of color 4-7 occurs when the ratio of the gap over the chemical potential mismatch, $\Delta / \delta \mu$, decreases below a value $\sqrt{2}$. Resolving the chromomagnetic instability and clarifying

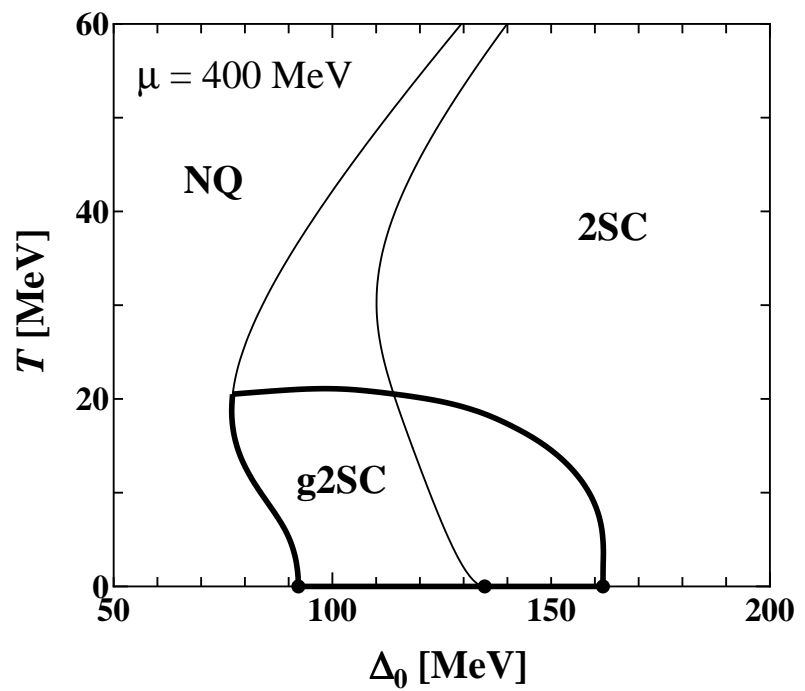

FIG. 1: The phase diagram of electrically neutral two-flavor quark matter in the plane of $\Delta_{0}$ and $T$. At $T=0$, the g2SC phase exists in the window $92 \mathrm{MeV}<\Delta_{0}<134 \mathrm{MeV}$ and the $2 \mathrm{SC}$ window is given by $\Delta_{0}>134 \mathrm{MeV}$. The unstable region for gluons 4-7 is depicted by the region enclosed by the thick solid line. The g2SC phase and a part of the $2 \mathrm{SC}$ phase $\left(92 \mathrm{MeV}<\Delta_{0}<162 \mathrm{MeV}\right)$ suffer from the chromomagnetic instability at $T=0$. The quark chemical potential is taken to be $\mu=400 \mathrm{MeV}$.

*Electronic address: kiriyama@th.physik.uni-frankfurt.de 
the nature of true ground state of dense quark matter are central issues in the study of color superconductivity 16, 17, 18, 19, 20, 21, 22, 23, 24, 25, 26, 27, 28, 29, 30, 31, 32, 33, 34, 35, 36, 37]. (For a three-flavor case, see Refs. [38, 39, 40, 41, 42, 43, 44.)

As an example, in Fig. 11, we plot the phase diagram of neutral two-flavor quark matter in the plane of the 2SC gap at $\delta \mu=0\left(\Delta_{0}\right)$ and temperature $(T)\left[31,32\right.$. (The parameter $\Delta_{0}$ is essentially the diquark coupling strength.) In order to obtain this diagram, we employed a gauged Nambu-Jona-Lasinio (NJL) model, which is the very same model that we shall use in this paper. Furthermore, we neglected the color chemical potential. The 2SC/g2SC phases and the unpaired normal quark (NQ) phase were included in the analysis. The quark chemical potential was taken to be $\mu=400 \mathrm{MeV}$, which is a value typical for the cores of compact stars. The region enclosed by the thick solid line is unstable (because gluons of color 4-7 have tachyonic Meissner masses there) and, therefore, should be replaced by other chromomagnetically stable phases, for instance, gluonic phases [24, 25]. (For more detailed discussions of the gluonic phases, see Refs. [45, 46].) Note, however, that we did not consider the global structure of a free energy in extracting the unstable region, but only the tendency toward the vector condensation $\left\langle\overrightarrow{A^{6}}\right\rangle$ in the $2 \mathrm{SC} / \mathrm{g} 2 \mathrm{SC}$ phases. A self-consistent analysis of the gluonic phases at $T=0$ has recently been done by Hashimoto and Miransky [36] and they found that the gluonic phase (strictly speaking, the gluonic cylindrical phase II) exists in the window $65 \mathrm{MeV}<\Delta_{0}<160 \mathrm{MeV}$ and is energetically more favored than the $2 \mathrm{SC} / \mathrm{g} 2 \mathrm{SC} / \mathrm{NQ}$ phases in this whole window.

In this paper, we study the gluonic cylindrical phase II at nonzero temperature and revisit the phase diagram shown in Fig. 1, computing the free energy of the gluonic phase in a self-consistent manner. The result would be useful for the phase diagram of QCD, and the compact star phenomenology as well.

\section{MODEL}

In order to study the gluonic phase, we use the gauged NJL model with massless up and down quarks:

$$
\mathcal{L}=\bar{\psi}\left(i \not D+\hat{\mu} \gamma^{0}\right) \psi+G_{D}\left(\bar{\psi} i \gamma_{5} \varepsilon \epsilon^{b} C \bar{\psi}^{T}\right)\left(\psi C i \gamma_{5} \varepsilon \epsilon^{b} \psi\right)-\frac{1}{4} F_{\mu \nu}^{a} F^{a \mu \nu}
$$

where the quark field $\psi$ carries flavor $\left(i, j=1, \ldots N_{f}\right.$ with $\left.N_{f}=2\right)$ and color $\left(\alpha, \beta=1, \ldots N_{c}\right.$ with $\left.N_{c}=3\right)$ indices, $C$ is the charge conjugation matrix; $(\varepsilon)^{i k}=\varepsilon^{i k}$ and $\left(\epsilon^{b}\right)^{\alpha \beta}=\epsilon^{b \alpha \beta}$ are the antisymmetric tensors in flavor and color spaces, respectively. The diquark coupling strength in the scalar $\left(J^{P}=0^{+}\right)$color-antitriplet channel is denoted by $G_{D}$. The covariant derivative and the field strength tensor are defined as

$$
\begin{aligned}
D_{\mu} & =\partial_{\mu}-i g A_{\mu}^{a} T^{a}, \\
F_{\mu \nu}^{a} & =\partial_{\mu} A_{\nu}^{a}-\partial_{\nu} A_{\mu}^{a}+g f^{a b c} A_{\mu}^{b} A_{\nu}^{c} .
\end{aligned}
$$

To evaluate loop diagrams we use a three-momentum cutoff $\Lambda=653.3 \mathrm{MeV}$ throughout this paper. In NJL-type models without dynamic gauge fields, one has to introduce color and electric chemical potentials $\left(\mu_{8}\right.$ and $\left.\mu_{e}\right)$ by hand [47] to ensure color- and electric-charge neutrality. In $\beta$-equilibrated neutral two-flavor quark matter, the elements of the diagonal matrix of quark chemical potentials $\hat{\mu}$ are given by

$$
\begin{aligned}
\mu_{u r} & =\mu_{u g}=\bar{\mu}-\delta \mu, \\
\mu_{d r} & =\mu_{d g}=\bar{\mu}+\delta \mu, \\
\mu_{u b} & =\bar{\mu}-\delta \mu-\mu_{8}, \\
\mu_{d b} & =\bar{\mu}+\delta \mu-\mu_{8},
\end{aligned}
$$

with

$$
\bar{\mu}=\mu-\frac{\delta \mu}{3}+\frac{\mu_{8}}{3}, \delta \mu=\frac{\mu_{e}}{2} .
$$

In Nambu-Gor'kov space, the inverse full quark propagator $S^{-1}(p)$ is written as

$$
S^{-1}(p)=\left(\begin{array}{cc}
\left(S_{0}^{+}\right)^{-1} & \Phi^{-} \\
\Phi^{+} & \left(S_{0}^{-}\right)^{-1}
\end{array}\right)
$$

with

$$
\begin{aligned}
& \left(S_{0}^{+}\right)^{-1}=\gamma^{\mu} p_{\mu}+\left(\bar{\mu}-\delta \mu \tau^{3}\right) \gamma^{0}+g \gamma^{\mu} A_{\mu}^{a} T^{a} \\
& \left(S_{0}^{-}\right)^{-1}=\gamma^{\mu} p_{\mu}-\left(\bar{\mu}-\delta \mu \tau^{3}\right) \gamma^{0}-g \gamma^{\mu} A_{\mu}^{a} T^{a T}
\end{aligned}
$$


and

$$
\Phi^{-}=-i \varepsilon \epsilon^{b} \gamma_{5} \Delta, \Phi^{+}=-i \varepsilon \epsilon^{b} \gamma_{5} \Delta .
$$

Here $\tau^{3}=\operatorname{diag}(1,-1)$ is a matrix in flavor space. Following the usual convention, we have chosen the diquark condensate to point in the third direction in color space.

For the gluonic cylindrical phase II, $B=\left\langle g A_{z}^{6}\right\rangle$ is the most relevant condensate, because the chromomagnetic instability related to gluons $4-7$ corresponds to the tachyonic mode in the direction of $B .24$, 25, 30] Besides $B$, we have to introduce a color chemical potential $\mu_{3}=\left\langle g A_{0}^{3}\right\rangle$ to ensure color neutrality at $B \neq 0$. Taking into account these condensates, the free energy of the gluonic phase in the one-loop approximation is given by

$$
\begin{aligned}
\Omega\left(\Delta, \mu_{e}, \mu_{8}, B, \mu_{3} ; \mu, T\right) \\
=-\frac{\mu_{8}^{2} B^{2}}{2 g^{2}}+\frac{\mu_{3} \mu_{8} B^{2}}{2 g^{2}}-\frac{\mu_{3}^{2} B^{2}}{8 g^{2}} \\
\quad-\frac{1}{12 \pi^{2}}\left(\mu_{e}^{4}+2 \pi^{2} T^{2} \mu_{e}^{2}+\frac{7 \pi^{4}}{15} T^{4}\right) \\
+\frac{\Delta^{2}}{4 G_{D}}-\frac{1}{2} \sum_{a} \int \frac{d^{3} p}{(2 \pi)^{3}}\left[\left|\epsilon_{a}\right|+2 T \ln \left(1+e^{-\beta\left|\epsilon_{a}\right|}\right)\right],
\end{aligned}
$$

where $\beta=1 / T$, the $\epsilon_{a}$ 's are quasi-quark energies and the sum runs over all particle and anti-particle $\epsilon_{a}$ 's. Here, we added tree-level contributions from gluons (first line on the r.h.s.),

$$
\Omega_{g}^{\text {(tree) }}=\frac{g^{2}}{4} f^{a b c} f^{a d e} A_{\mu}^{b} A_{\nu}^{c} A^{d \mu} A^{e \nu},
$$

and electrons (second line on the r.h.s.). Note also that the $\epsilon_{a}$ 's depend on the vector condensates through the covariant derivatives in the quark propagator (5). In what follows, we neglect the color chemical potentials $\mu_{3,8}$ and, consequently, the tree-level contributions of gluons. We have carefully checked that their effect on the free energy is negligible for realistic values of $\alpha_{s} \simeq 1$ (see also Ref. [36]).

In this work, in order to remove the ultraviolet divergence in the Meissner screening masses we shall use the following subtraction

$$
\Omega_{R}=\Omega\left(\Delta, \mu_{e}, B ; \mu, T\right)-\Omega(0,0, B ; 0,0) .
$$

It is known that this free energy subtraction is not adequate to remove the cutoff dependence of the free energy at $T>0$. In fact, Eq. (10) leads to positive Meissner screening masses in the normal phase at $T>0$ [31, 32]. In this work we do not go into this problem because this unphysical behavior of the Meissner masses is nothing but a cutoff artifact and moreover is negligibly small at $\mu=400 \mathrm{MeV}$ and at the temperatures of interest (20 MeV at most).

In order to find the neutral gluonic phase, we first solve a set of coupled equations (the gap equation and the electrical charge neutrality condition),

$$
\frac{\partial \Omega_{R}}{\partial \Delta}=\frac{\partial \Omega_{R}}{\partial \mu_{e}}=0,
$$

as a function of $B$ and, then, compute the free energy $\Omega_{R}(B)$. Finally, the minimum of $\Omega_{R}(B)$ determines the neutral gluonic phase. (In the following Figs. 3 and 4 , we plot the free energy evaluated along the solution of the coupled equations (11).)

\section{NUMERICAL RESULTS}

Figure 2 shows $\Delta, \delta \mu$ and $B$ in the gluonic phase at $T=0$ as a function of $\Delta_{0}$. First, let us note that the results of Fig. 2 are in good agreement with those shown in Figs. 1, 2, and 3 of Ref. 36], where the color chemical potentials $\mu_{3,8}$ were treated self-consistently. In Fig. 2 one can see that the gluonic phase exists in the window

$$
66 \mathrm{MeV}<\Delta_{0}<162 \mathrm{MeV} .
$$

The gluonic phase is energetically favored over the $2 \mathrm{SC} / \mathrm{g} 2 \mathrm{SC} / \mathrm{NQ}$ phases in this whole window (see also Fig. 5 of Ref. [36]). One also sees that the phase transition between the gluonic phase and the NQ (2SC) phase at $\Delta_{0}=66 \mathrm{MeV}$ $(162 \mathrm{MeV})$ is strongly (weakly) of first order. 


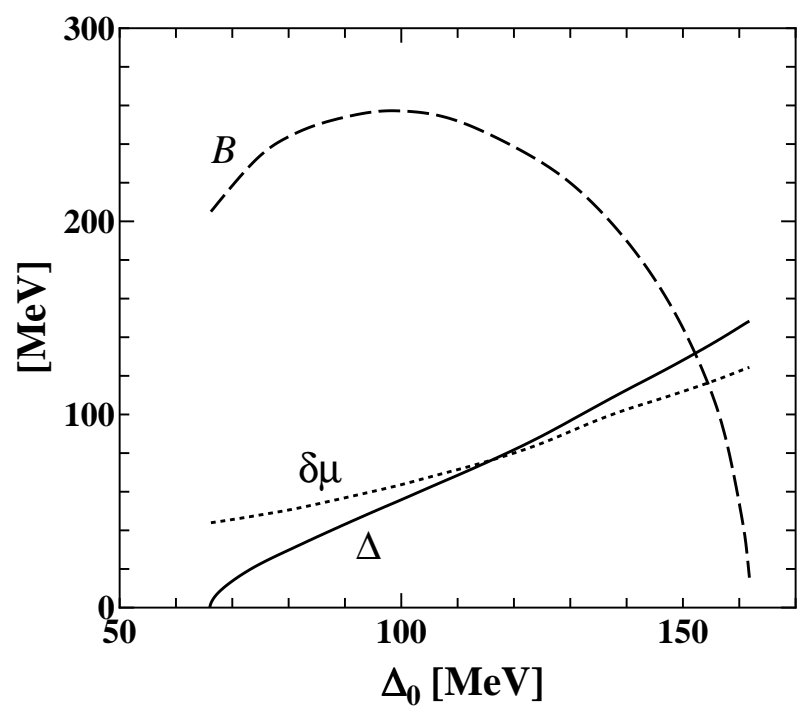

FIG. 2: The gap parameter $\Delta$ (solid line), the chemical potential mismatch $\delta \mu$ (dotted line) and the gluonic vector condensate $B$ (dashed line) versus $\Delta_{0}$ in the gluonic phase at $T=0$. The quark chemical potential is taken to be $\mu=400 \mathrm{MeV}$.

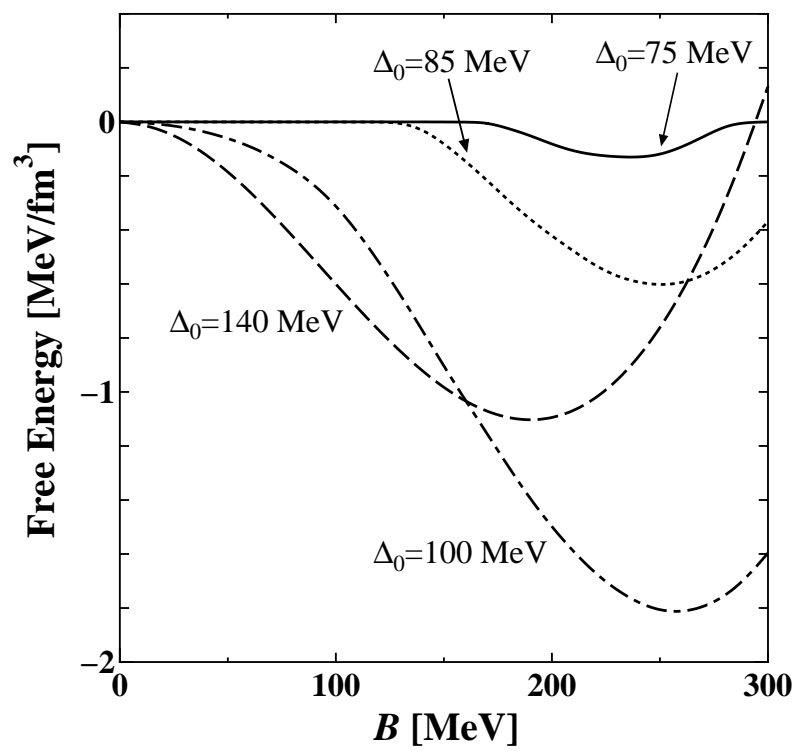

FIG. 3: The free energy $\Omega_{R}(B)$ as a function of $B$ at $T=0$ for $\Delta_{0}=75 \mathrm{MeV}$ (solid line), $\Delta_{0}=85 \mathrm{MeV}$ (dotted line), $\Delta_{0}=100 \mathrm{MeV}$ (dot-dashed line), and $\Delta_{0}=140 \mathrm{MeV}$ (dashed line). Note that the free energy is measured with respect to the 2SC/g2SC/NQ phases at $B=0$. The results are plotted for $\mu=400 \mathrm{MeV}$.

Now let us take a closer look at the free energy at $T=0$. Figure 3 shows the behavior of $\Omega_{R}(B)$ measured with respect to the $2 \mathrm{SC} / \mathrm{g} 2 \mathrm{SC} / \mathrm{NQ}$ phases at $B=0$. The results are plotted for $\mu=400 \mathrm{MeV}$ at several values of $\Delta_{0}$.

In the weak coupling regime, $66 \mathrm{MeV}<\Delta_{0}<92 \mathrm{MeV}$, the chromomagnetic instability does not exist in the NQ phase (see Fig. 1). We note that the curvature of $\Omega_{R}(B)$ at $B=0$,

$$
m_{M}^{2}=\left.\frac{d^{2} \Omega_{R}(B)}{d B^{2}}\right|_{B=0}
$$

can be regarded as the Meissner mass squared $\partial^{2} \Omega_{R} /\left.\partial B^{2}\right|_{B=0}$ in the 2SC/g2SC/NQ phases, since the solutions of Eq. (11) satisfy $\Delta=\bar{\Delta}+\mathcal{O}\left(B^{2}\right)$ and $\mu_{e}=\bar{\mu}_{e}+\mathcal{O}\left(B^{2}\right)$ for small values of $B$, where $\bar{\Delta}$ and $\bar{\mu}_{e}$ denote their values at $B=0\left[24,[25]\right.$. We found that $m_{M}^{2}$ is indeed zero in the weak coupling regime. In addition, we observed that, for small values of $B$, the system is in the ungapped $(\Delta=0)$ phase and the free energy behaves like $\Omega_{R} \sim \mathcal{O}\left(B^{4}\right)$. 

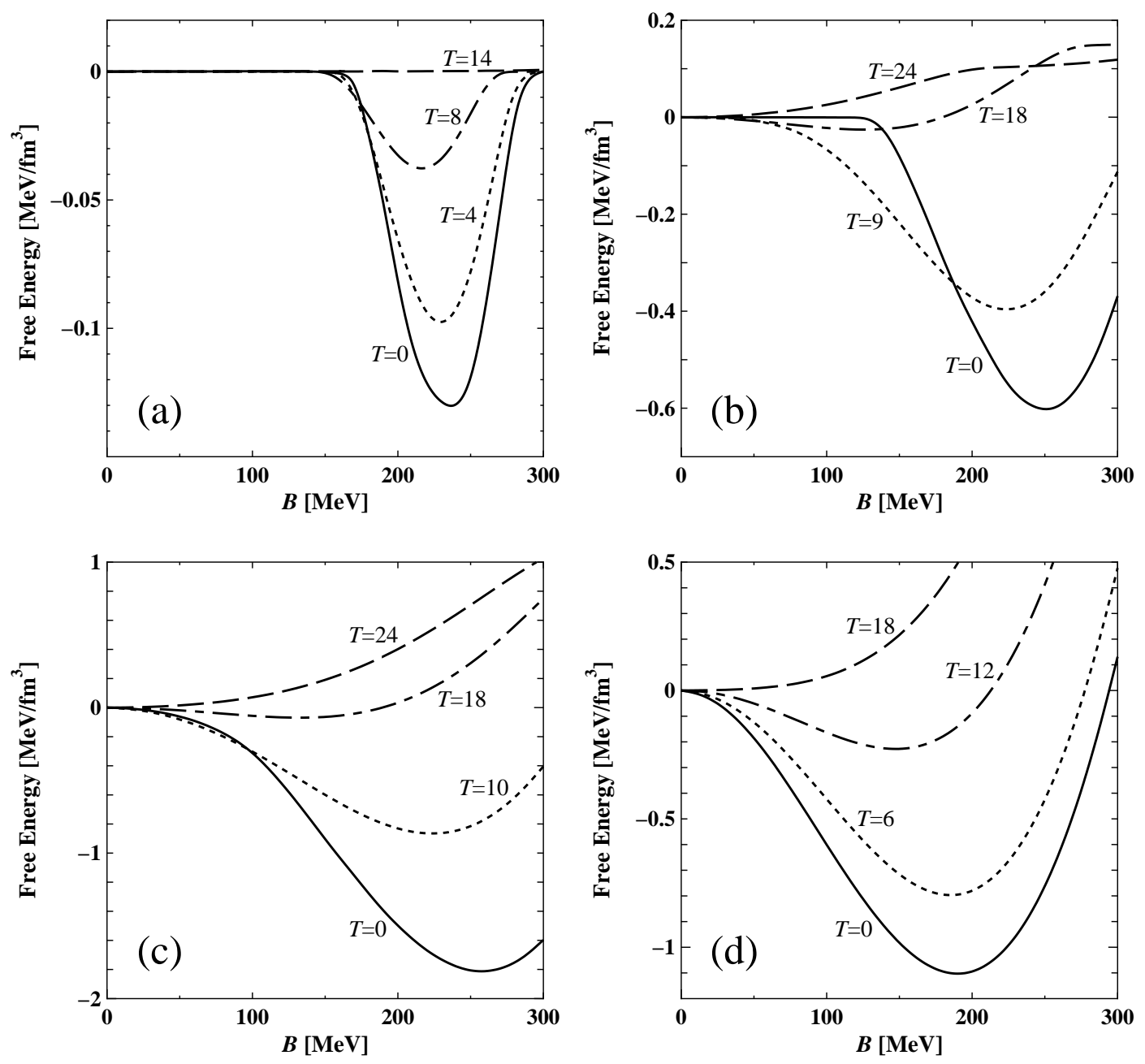

FIG. 4: The temperature dependence of the free energy (measured with respect to the $2 \mathrm{SC} / \mathrm{g} 2 \mathrm{SC} / \mathrm{NQ}$ phases at $B=0$ ) as a function of $B$ for $\Delta_{0}=75 \mathrm{MeV}$ (a), for $\Delta_{0}=85 \mathrm{MeV}$ (b), for $\Delta_{0}=100 \mathrm{MeV}$ (c), and for $\Delta_{0}=140 \mathrm{MeV}$ (d). The results are plotted for $\mu=400 \mathrm{MeV}$ and the values of $T$ are given in $\mathrm{MeV}$.

However, contrary to the result of Fig. 1 the free energy has a global minimum at $B \neq 0$ and the gluonic phase is energetically favored over the NQ phase. For $92 \mathrm{MeV}<\Delta_{0} \lesssim 162 \mathrm{MeV}$, one finds tachyonic modes at $B=0$ because the g2SC phase and a part of the 2SC phase suffer from the chromomagnetic instability and, therefore, are unstable against the formation of $B$. Consequently, the gluonic phase is realized in this region, as expected. For strong coupling, $\Delta_{0} \gtrsim 162 \mathrm{MeV}$, the $2 \mathrm{SC}$ phase is chromomagnetically stable in this regime and the free energy has a global minimum at $B=0$, though it is not plotted in Fig. 3.

We now turn to the free energy of the gluonic phase at $T>0$. Figure 4 (a) display the temperature dependence of the free energy for $\Delta_{0}=75 \mathrm{MeV}$. As $T$ grows, the free-energy gain gets reduced, but the change of the vacuum expectation value of $B$ is rather small. As a result, we observe a strong first-order transition from the gluonic phase to the NQ phase at $T \simeq 14 \mathrm{MeV}$. Note that $m_{M}^{2}$ remains positive at any value of $T$, which is consistent with the result shown in Fig. [1.

In Fig. 四(b), the same plot is displayed for $\Delta_{0}=85 \mathrm{MeV}$. At low temperature, like in the case of $\Delta_{0}=75 \mathrm{MeV}$, the gluonic phase is more favored than the chromomagnetically stable NQ phase. At $T \simeq 9 \mathrm{MeV}, m_{M}^{2}$ turns negative, meaning that the stable NQ phase undergoes a phase transition into the unstable g2SC phase (see Fig. 11). The gluonic phase is energetically favored until the temperature reaches $T \simeq 20 \mathrm{MeV}$. Above this temperature, the g2SC phase becomes stable and therefore is favored.

In Figs. 4(c) and 4(d), we plot the free energy for the cases of $\Delta_{0}=100 \mathrm{MeV}$ and $\Delta_{0}=140 \mathrm{MeV}$, respectively. In both cases, the gluonic phase is energetically favored at low temperature. In contrast, at high temperature, the global minima of the free energy are realized at $B=0$, which means that, as expected from the result of Fig. 1, the 


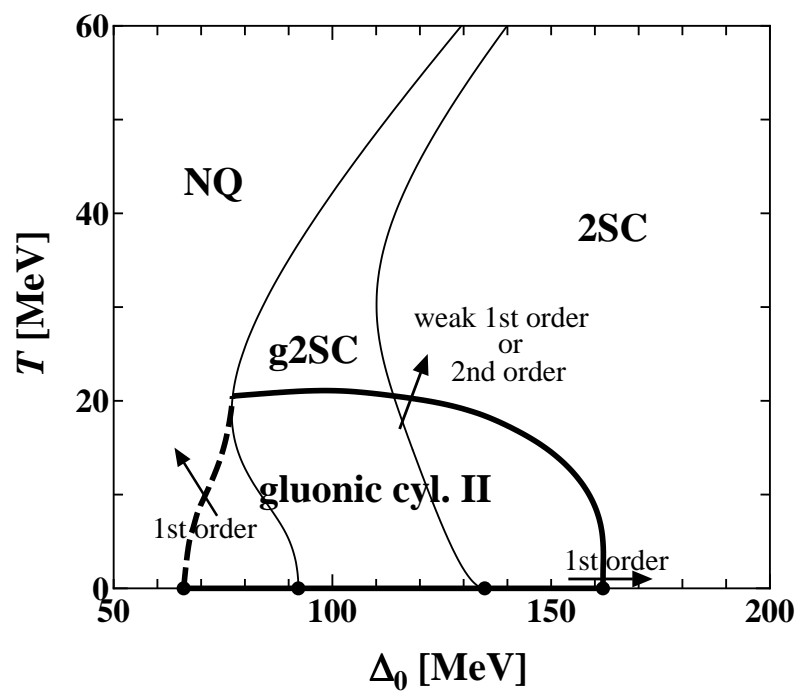

FIG. 5: Schematic phase diagram of neutral two-flavor quark matter at moderate density in $\Delta_{0}-T$ plane. The thick solid line denotes the line of second-order or weakly first-order transitions and strong first-order transitions are indicated by a thick dashed line. In the region enclosed by the thick solid and dashed lines, the gluonic phase is energetically more favored than the $2 \mathrm{SC} / \mathrm{g} 2 \mathrm{SC} / \mathrm{NQ}$ phases.

chromomagnetically stable $2 \mathrm{SC} / \mathrm{g} 2 \mathrm{SC}$ phases are favored. For $\Delta_{0}=100 \mathrm{MeV}$, the phase transition from the gluonic phase to the g2SC phase takes place at $T \simeq 21 \mathrm{MeV}$. In the case of $\Delta_{0}=140 \mathrm{MeV}$, the phase transition takes place at $T \simeq 18 \mathrm{MeV}$.

Here, we would like to make a comment regarding the order of the phase transitions. As mentioned above, we observed the strong first-order transition (gluonic phase $\leftrightarrow$ NQ phase) at $\Delta_{0}=75 \mathrm{MeV}$. On the other hand, for the cases of $\Delta_{0}=85,100,140 \mathrm{MeV}$, the phase transition (gluonic phase $\leftrightarrow 2 \mathrm{SC} / \mathrm{g} 2 \mathrm{SC}$ phases) is likely to be of second order. However, evaluating the free energy self-consistently near the critical temperatures is not easy and hence we do not exclude the possibility of weak first-order transitions. Furthermore, it should be also mentioned that, because of the cutoff artifact in Eq. (10), it might be impossible to distinguish a weak first-order transition from a second-order one.

\section{SUMMARY, CONCLUSIONS, AND OUTLOOK}

\section{A. Phase diagram}

We studied the gluonic cylindrical phase II at nonzero temperature. Using the gauged NJL model and the one-loop approximation, we computed the free energy of the gluonic phase self-consistently and investigated the phase structure of the gluonic phase. Although we neglected the color chemical potentials, we have checked that, for $\alpha_{s} \simeq 1$, their effect on the free energy is negligible.

In the weak coupling regime, we found that the gluonic phase undergoes a strong first-order transition into the NQ phase as it is heated. This is a new aspect of the gluonic phase at $T>0$, which is not shown in Fig. 1, On the other hand, since the phase transitions from the gluonic phase to the chromomagnetically stable $2 \mathrm{SC} / \mathrm{g} 2 \mathrm{SC}$ phases are of second-order or weakly first-order, we expect that the corresponding critical line shown in Fig. 11(i.e., the right branch of the thick solid line) is not drastically altered by the self-consistent analysis. (In other words, the Meissner masses squared can be a rough criterion for choosing the energetically favored phase in this regime.) We thus are able to make a sketch of a schematic phase diagram of two-flavor quark matter, which is free from the chromomagnetic instability related to gluons 4-7 (see Fig. 5). ${ }^{1}$ The low-temperature region of the g2SC phase and a part of the 2SC phase is replaced by the gluonic phase. Furthermore, the gluonic phase wins against a part of the NQ phase and

\footnotetext{
${ }^{1}$ It is interesting to note that the neutral single plane-wave Larkin-Ovchinnikov-Fulde-Ferrell state [48, 49] has a similar phase structure as the gluonic phase [26, 31, 32, 34].
} 

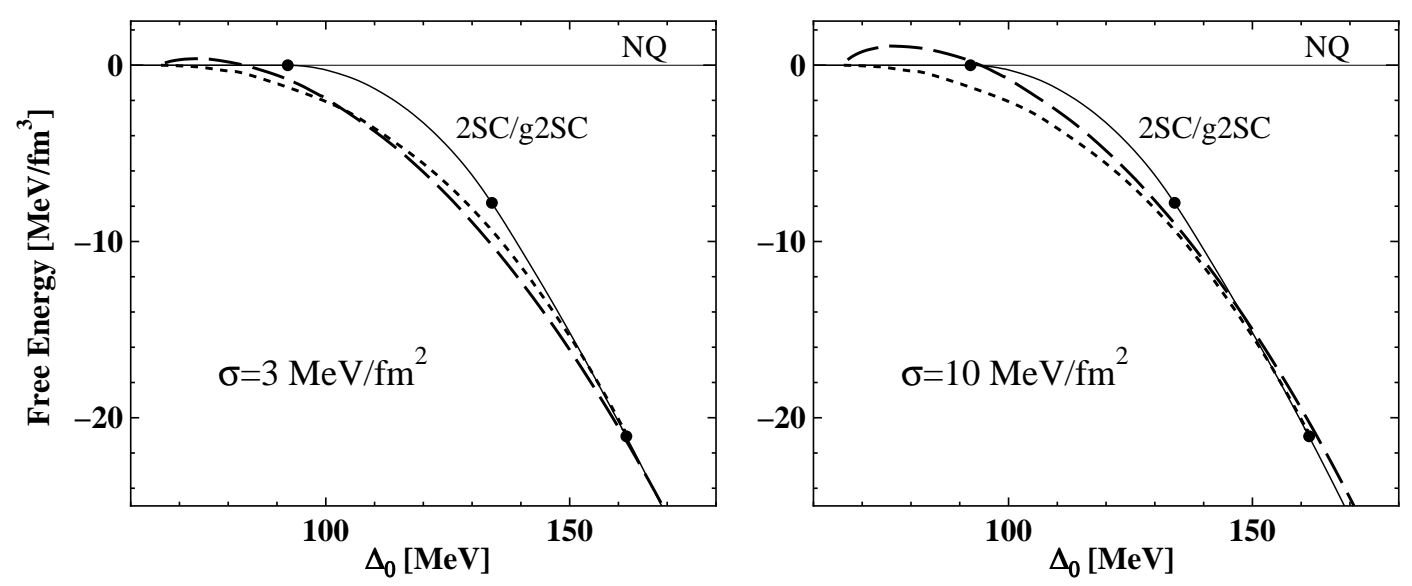

FIG. 6: The free energy of the neutral 2SC/g2SC phase (solid line), the gluonic phase (dotted line), and the mixed phase (dashed line) measured with respect to the NQ phase as a function of $\Delta_{0}$ for $\sigma=3 \mathrm{MeV} / \mathrm{fm}^{2}$ (left) and $\sigma=10 \mathrm{MeV} / \mathrm{fm}^{2}$ (right). The three dots on the solid line $\left(\Delta_{0}=92,134,162 \mathrm{MeV}\right.$ from left to right) denote the edge of the g2SC window with the normal phase, the phase transition point between the 2SC and the g2SC phases, and the critical point of the chromomagnetic instability. The quark chemical potential is taken to be $\mu=400 \mathrm{MeV}$.

enlarges its region. We argue therefore that the gluonic phase which could resolve the chromomagnetic instability related to gluons $4-7$ is a strong candidate for the ground state of a neutral two-flavor color superconductor in the intermediate coupling regime. Alternatives include other types of the gluonic phases [24, 25, 36, 37], the crystalline phases [10, 11, 43] and the mixed phase [19]. It should be mentioned that, at $T=0$, the gluonic color-spin locked phase is more stable than the gluonic cylindrical phase II in some region of $\Delta_{0}$ and moreover is free from the chromomagnetic instability at moderate densities [54, 55].

Although we concentrated on the phase diagram in $T-\Delta_{0}$ plane in this work, it is obviously worthwhile to revisit the phase diagram in $T$ - $\mu$ plane. A preliminary study [50] indicates that currently known phase diagrams [51, 52, 53] must be significantly altered. In addition, the critical temperature for the gluonic phase could reach a few tens of $\mathrm{MeV}$ and, therefore, it is interesting to study astrophysical implications of the gluonic phase, e.g., the quark matter equation of state, neutrino emission from compact star cores, and so on.

\section{B. Gluonic phase versus mixed phase}

Finally we briefly look at a mixed phase consisting of the NQ and the 2SC phases [19, 20, 21]. For the mixed phase to exist, it must satisfy the Gibbs conditions, which are equivalent to chemical and mechanical equilibrium conditions between the NQ and the 2SC phases. These conditions end up as follows

$$
P^{(\mathrm{NQ})}\left(\mu, \mu_{e}\right)=P^{(2 \mathrm{SC})}\left(\mu, \mu_{e}\right) .
$$

Beside Eq. (14) two components must have opposite electrical charge densities. Otherwise a globally neutral mixed phase could not exist. We solved Eq. (14) and found that the globally neutral mixed phase exists in the window

$$
67 \mathrm{MeV}<\Delta_{0}<201 \mathrm{MeV},
$$

where the quark chemical potential was taken to be $\mu=400 \mathrm{MeV}$.

In order to calculate the free energy of the mixed phase we take account of finite-size effects, i.e., the surface and Coulomb energies associated with phase separation. The surface and Coulomb energy densities are given by

$$
\epsilon_{S}=\frac{d x \sigma}{r_{0}}, \epsilon_{C}=2 \pi \alpha_{\mathrm{em}} f_{d}(x) x\left(\Delta n_{e}\right)^{2} r_{0}^{2},
$$

where $\sigma$ is the surface tension, $x$ is the volume fraction of the rarer phase, $\Delta n_{e}$ is the difference of the electric charge density between $\mathrm{NQ}$ and 2SC phases, and $\alpha_{\mathrm{em}}=1 / 137$. These energy densities also depend on the dimension $d$ ( $d=1,2$, and 3 correspond to slabs, rods, and droplets configurations, respectively) and $r_{0}$, which denotes the radius of the rarer phase. The geometrical factor $f_{d}(x)$ is given by

$$
f_{d}(x)=\frac{1}{d+2}\left(\frac{2-d x^{1-2 / d}}{d-2}+x\right) .
$$


Minimizing the sum of $\epsilon_{S}$ and $\epsilon_{C}$ with respect to $r_{0}$, we obtain

$$
\epsilon_{S}+\epsilon_{C}=\frac{3}{2}\left(4 \pi \alpha_{\mathrm{em}} d^{2} f_{d}(x) x^{2}\right)^{1 / 3}\left(\Delta n_{e}\right)^{2 / 3} \sigma^{2 / 3} .
$$

The actual value of the surface tension in quark matter is poorly known, in this work we assume $d=3$ (droplets configuration) and try relatively small surface tension.

Figure 6 displays the free energy of the 2SC/g2SC phase, the gluonic phase, and the mixed phase. For a very small surface tension $\sigma=3 \mathrm{MeV} / \mathrm{fm}^{2}$, the mixed phase is the most favored in a wide range of $\Delta_{0}, 103 \mathrm{MeV}<\Delta_{0}<166 \mathrm{MeV}$. The gluonic phase is energetically more favored than the mixed phase only in the weak coupling regime. Note that the value of the surface tension, $\sigma=3 \mathrm{MeV} / \mathrm{fm}^{2}$ at $\mu=400 \mathrm{MeV}$, is close to that calculated by Reddy and Rupak [19]. For a surface tension $\sigma=10 \mathrm{MeV} / \mathrm{fm}^{2}$, there still is a wide window where the mixed phase is more stable than the g2SC phase, but the mixed phase is less favored than the gluonic phase.

It should be mentioned here that, however, we did not take into account the thickness of the boundary layer, which has been estimated to be comparable to the value of the Debye screening length in each of the two phases, and therefore the results shown in Fig. 6] is not a final conclusion [56, 57]. The effect of charge screening would increase the surface energy substantially [58, 59].

\section{Acknowledgments}

I would like to thank Dirk Rischke and Armin Sedrakian for discussions and for comments on the earlier version of the manuscript. I also would like to thank H. Abuki, M. Ruggieri, and I. Shovkovy for discussions during the YITP international symposium "Fundamental Problems in Hot and/or Dense QCD". This work was supported by the Deutsche Forschungsgemeinschaft (DFG).

[1] K. Rajagopal and F. Wilczek, in At the Frontier of Particle Physics/Handbook of QCD, edited by M. Shifman (World Scientific, Singapole, 2001).

[2] M. G. Alford, Annu. Rev. Nucl. Part. Sci. 51, 131 (2001).

[3] D. K. Hong, Acta. Phys. Pol. B 32, 1253 (2001).

[4] S. Reddy, Acta. Phys. Pol. B 33, 4101 (2002).

[5] D. H. Rischke, Prog. Part. Nucl. Phys. 52, 197 (2004).

[6] R. Casalbuoni and G. Nardulli, Rev. Mod. Phys. 76, 263 (2004).

[7] M. Buballa, Phys. Rept. 407, 205 (2005).

[8] M. Huang, Int. J. Mod. Phys. E 14, 675 (2005).

[9] I. A. Shovkovy, Found. Phys. 35, 1309 (2005).

[10] M. Alford, J. A. Bowers, and K. Rajagopal, Phys. Rev. D 63, 074016 (2001).

[11] J. A. Bowers and K. Rajagopal, Phys. Rev. D 66, 065002 (2002).

[12] I. Shovkovy and M. Huang, Phys. Lett. B 564, 205 (2003).

[13] M. Huang and I. Shovkovy, Nucl. Phys. A729, 835 (2003).

[14] M. Huang and I. A. Shovkovy, Phys. Rev. D 70, 051501(R) (2004).

[15] M. Huang and I. A. Shovkovy, Phys. Rev. D 70, 094030 (2004).

[16] I. Giannakis and H. C. Ren, Phys. Lett. B 611, 137 (2005).

[17] I. Giannakis and H. C. Ren, Nucl. Phys. B723, 255 (2005).

[18] I. Giannakis, D. f. Hou, and H. C. Ren, Phys. Lett. B 631, 16 (2005).

[19] S. Reddy and G. Rupak, Phys. Rev. C 71, 025201 (2005).

[20] F. Neumann, M. Buballa, M. Oertel, Nucl. Phys. A714, 481 (2003).

[21] I. Shovkovy, M. Hanauske, M. Huang, Phys. Rev. D 67, 103004 (2003).

[22] M. Huang, Phys. Rev. D 73, 045007 (2006).

[23] D. K. Hong, hep-ph/0506097.

[24] E. V. Gorbar, M. Hashimoto, and V. A. Miransky, Phys. Lett. B 632, 305 (2006).

[25] E. V. Gorbar, M. Hashimoto, and V. A. Miransky, Phys. Rev. D 75, 085012 (2007).

[26] E. V. Gorbar, M. Hashimoto, and V. A. Miransky, Phys. Rev. Lett. 96, 022005 (2006).

[27] K. Fukushima, Phys. Rev. D 73, 094016 (2006).

[28] E. V. Gorbar, M. Hashimoto and V. A. Miransky, and I. A. Shovkovy, Phys. Rev. D 73, 111502(R) (2006).

[29] M. Hashimoto, Phys. Lett. B 642, 93 (2006).

[30] O. Kiriyama, D. H. Rischke, and I. A. Shovkovy, Phys. Lett. B 643, 331 (2006).

[31] O. Kiriyama, Phys. Rev. D 74, 074019 (2006).

[32] O. Kiriyama, Phys. Rev. D 74, 114011 (2006). 
[33] K. Iida and K. Fukushima, Phys. Rev. D 74, 074020 (2006).

[34] L. He, M. Jin, and P. Zhuang, Phys. Rev. D 75, 036003 (2007).

[35] R. Gatto and M. Ruggieri, Phys. Rev. D 75, 114004 (2007).

[36] M. Hashimoto and V. A. Miransky, Prog. Theor. Phys. 118, 303 (2007).

[37] E. J. Ferrer and V. de la Incera, Phys. Rev. D 76, 045011 (2007).

[38] R. Casalbuoni, R. Gatto, M. Mannarelli, G. Nardulli, and M. Ruggieri, Phys. Lett. B 605, 362 (2005); 615, 297(E) (2005).

[39] K. Fukushima, Phys. Rev. D 72, 074002 (2005).

[40] A. Kryjevski, hep-ph/0508180

[41] T. Schäfer, Phys. Rev. Lett. 96, 012305 (2006).

[42] M. Ciminale, G. Nardulli, M. Ruggieri, and R. Gatto, Phys. Lett. B 636, 317 (2006).

[43] K. Rajagopal and R. Sharma, Phys. Rev. D 74, 094019 (2006).

[44] X. B. Zhang and J. I. Kapusta, Phys. Rev. D 75, 054012 (2007).

[45] E. V. Gorbar, J. Jia, and V. A. Miransky, Phys. Rev. D 73, 045001 (2006).

[46] A. Buchel, J. Jia, and V. A. Miransky, Nucl. Phys. B772, 323 (2007).

[47] M. Buballa and I. A. Shovkovy, Phys. Rev. D 72, 097501 (2005).

[48] A. I. Larkin and Y. N. Ovchinnikov, Zh. Eksp. Teor. Fiz. 47, 1136 (1964) [Sov. Phys. JETP 20, 762 (1965)].

[49] P. Fulde and R. A. Ferrell, Phys. Rev. 135, A550 (1964).

[50] O. Kiriyama, arXiv:0805.3304 [hep-ph].

[51] S. B. Rüster, V. Werth, M. Buballa, I. A. Shovkovy, and D. H. Rischke, Phys. Rev. D 72, 034004 (2005).

[52] D. Blaschke, S. Fredriksson, H. Grigorian, A. M. Öztaş, and F. Sandin, Phys. Rev. D 72, 065020 (2005).

[53] H. Abuki and T. Kunihiro, Nucl. Phys. A768, 118 (2006).

[54] M. Hashimoto and J. Jia, Phys. Rev. D 76, 114019 (2007).

[55] M. Hashimoto, Phys. Rev. D 78, 031501 (2008).

[56] H. Heiselberg, C. J. Pethick, E. F. Staubo, Phys. Rev. Lett. 70, 1355 (1993).

[57] N. K. Glendenning, S. Pei, Phys. Rev. C 52, 2250 (1995).

[58] T. Norsen and S. Reddy, Phys. Rev. C 63, 065804 (2001).

[59] D. N. Vorkresensky, M. Yasuhira, and T. Tatsumi, Nucl. Phys. A723, 291 (2003). 